\title{
The Bowl of Integers and the Hexlet*
}

\section{By Prof. Frederick Soddy, F.R.S.}

NTO a hollow sphere of unit radius, with a curvature or bend, therefore, -1 , put two solid spheres of radius $\frac{1}{2}$, or bend +2 . The two solid. spheres then kiss each other at the exact centre of since it has the unique property that the bend of every sphere of the infinitely infinite number of spheres that theoretically can be packed into it, so that each is located by its neighbours, is an exact

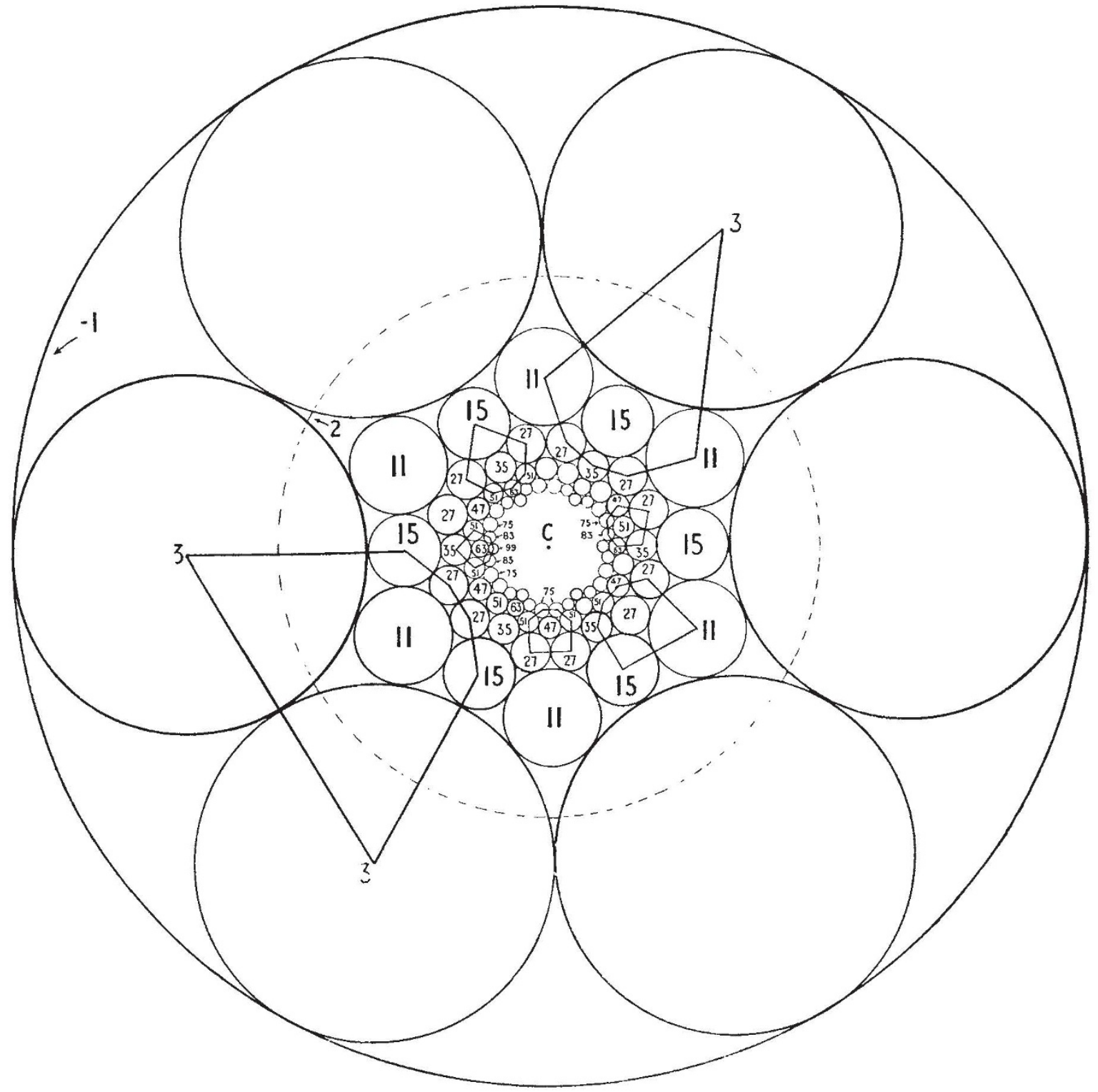

Fig. 1.

the bowl, and kiss the latter at the extremities of its diameter through all three centres, no other disposition being theoretically possible. This threesphere assembly may be termed the 'bowl of integers',

"Vide "The Kiss Precise" and "The Hexlet" (NATdre, 137, 1021, June 20, and 138, 958, Dreember 5, 1936). integer which can be written down at once from those of its neighbours. It enables the nature of the 'hexlet' to be elucidated without either trigonometry or the algebra of irrationals.

Fig. 1 shows a section through the bowl of integers through its centre, $C$, in the plane of the paper, 
which is also the point of contact of the two 2-spheres, one of which is above and one below it, the centres of the latter and their points of contact with the bowl being on the line through $C$ vertical to the plane of the paper. The circle shown by a dotted line is the 2-sphere below the plane of the paper, and the figures labelling the circles are the bends, or reciprocals of the radii, of the spheres centred in the plane of the paper, of which they are sections. The space between the two 2 -spheres and the bowl has been packed by successive rings of spheres, each ring in passing inwards consisting of ever larger and larger numbers of smaller and smaller spheres, until as we approach $C$ they become infinitely small and infinitely numerous. All are in contact with the two equal 2-spheres above and below the plane of the paper, and all, being centred in this plane, are accurately represented by circles. So wo may imagine

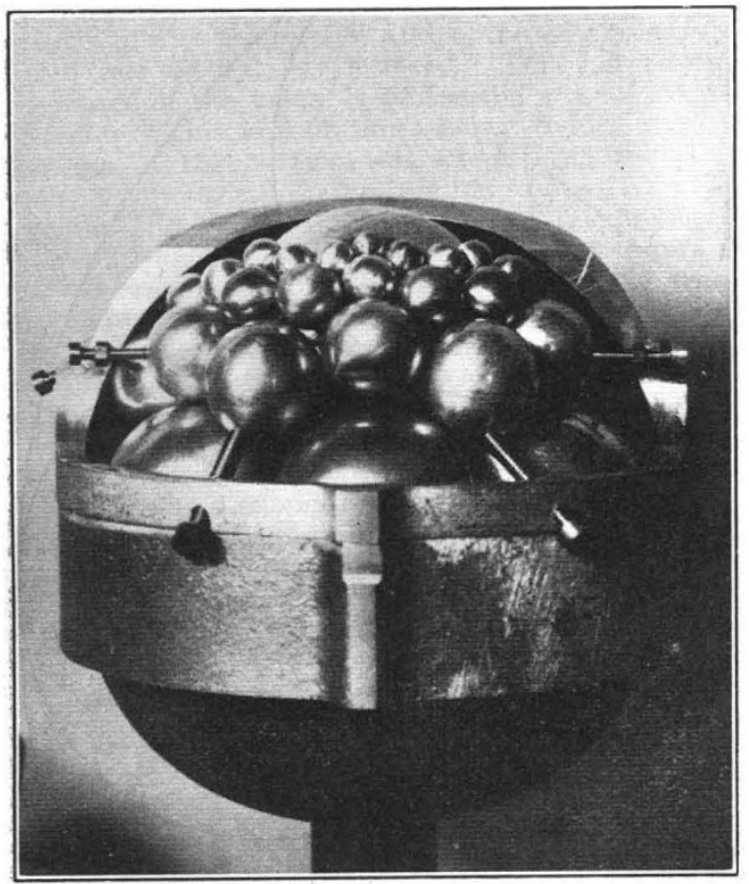

Fig. ?.

similar rings of spheres above and below the plane of the paper, each in contact with the bowl and with one of the two 2-spheres, and then begin all over again to fill up the remaining spaces and so on ad infinitum, every sphere added increasing the number that have to be added to fill it up !

These further spheres being spaced in three dimensions cannot be accurately represented by a drawing, but they are all characterized by exactly integral bends. I discovered this years ago for the simpler case of cylinders, or circles, in connexion with the design of an actual mechanism, but it is true also for the spherical analogue. So long as the sum of the two radii of the two largest equals that of the enclosure, the bends are always rational, and when the largest two are equal in radius, integral with respect to that of the enclosure as unit. So far as I am aware, this has not before been observed.

From the case illustrated, the principles of the hexlet are easily exhibited. The largest spheres that can be fitted in after the two of radius $1 / 2$ are a ring of six of radius $1 / 3$, the centres of which are at the corners of a regular hexagon. It is the simplest case of the hexlet; all six spheres being equal, the bend, 3 , is simply the sum of those of the common trio, namely, $2+2-1$. Going inward towards $C$ the next ring has twelve spheres, six 1l's alternating with six 15's; the next has 18, six contiguous pairs of 27's alternating with six single 35 's; the next twenty-four, the series, $63,51,47,51$ being six times repeated round the periphery; and the last to be shown in the diagram has thirty, the unit series being $75,83,99,83,75$.

Seven different hexlets belonging to the pair of 2 -spheres and, respectively, to the $11,15,27,35,47$, 51 and 63 spheres are indicated by the irregular hexagons formed by the six lines joining the centres of the hexlet. In every case the sum of the bends of any two opposite members is twice that of the central member plus 4 , the latter being the sum of the bends of the two 2 -spheres common to all the hexlets. Thus to take the last and smallest,

$$
99+35=83+51=51+83=2(63+2+2) .
$$

In the same way each of the six 3 -spheres is the centre of a hexlet, $-1,3,11,15,11,3$, opposite members of which sum to $2(3+2+2)$. Three being given, the other three may be written down without further calculation. The hexlet round the 27 - and 51 -spheres are unsymmetrical. The others exhibit three different types of symmetry.

Fig. 2 shows a model of the bowl of integers with the tangential hexlets of the upper half, each sphere being in contact with the bowl and upper of the two 2 -spheres. The unit series, six times repeated round the periphery, are for the successive rings I, 3 ; II, 6,5 ; III, $11,9,9$; IV, $18,15,14,15$; V, 27, $23,21,21,23$; VI, 38, 33, 30, 29, 30, 33 and so on. In complete generality, the hexlet derives as follows. As a corollary of Euclid (ii, props. 11 and 12) that the centres and point of contact of kissing circles are collinear, any three spheres can make mutual contact, all three centres being necessarily in one plane, forming a triangle any two sides of which are necessarily yreater than the third. But a fourth sphere may make mutual contact with them only if its size is between a maximum and minimum limit, which values apply when the contres are co-planar. Its size and position in space relatively to the others are dependent variables. The centre of the fourth, at its maximum say, may move out of the plane containing the centres of the trio, and the fourth sphere may retain contact with the trio anywhere in a closed orbit cutting the plane twice per revolution at the maximum and minimum, but only by shrinking or swelling in size between these two limits. These maximum and minimum values are those for the particular case when the hexlet round the trio is symmetrical (as in the hexlets in Fig. 1 round spheres 15,35 and 63), and is divided into symmetrical halves by the plane containing the three centres of the trio and the two of the opposite largest and smallest beads. These values are, since for this case the centres are co-planar, the two roots of the quadratic equation for four circles in mutual contact ("The Kiss Precise", verse 2):

$$
\begin{aligned}
& 2\left(\varphi^{2}+\alpha^{2}+\beta^{2}+\gamma^{2}\right)=(\varphi+\alpha+\beta+\gamma)^{2}, \\
& \text { or } \varphi \pm=\alpha+\beta+\gamma \pm 2 \sqrt{\alpha \beta+\alpha \gamma+\beta \gamma}
\end{aligned}
$$

where $\varphi$ stands for the bend of the fourth sphere and $\alpha, \beta$ and $\gamma$ for those of the trio, the sum of which 
will be termed $\sigma$. Equation (1) may be written $\varphi_{ \pm}=\sigma \pm 2 \rho$, where $\rho$ is the bend of the circle inscribed in the triangle formed by joining the centres of the trio. $\varphi_{+}+\varphi_{-}=2 \sigma$.

But if the fourth sphere has any other value, say $\delta$, between the maximum and minimum, the centres are no longer co-planar, but four bends being fixed, the fifth is determined by and can be found from the quadratic equation for five spheres in mutual contact (ibid., verse 3 ).

$3\left(\varphi^{2}+\alpha^{2}+\beta^{2}+\gamma^{2}+\delta^{2}\right)=(\varphi+\alpha+\beta+\gamma+\delta)^{2}$ or $\varphi \pm=\frac{1}{2}[\alpha+\beta+\gamma+\delta \pm\{6(\alpha \beta+\alpha \gamma+\alpha \delta+$ $\left.\left.\beta \gamma+\beta \delta+\gamma \delta)-3\left(\alpha^{2}+\beta^{2}+\gamma^{2}+\delta^{2}\right)\right\}^{1 / 2}\right]$

As always, we get two real roots, which refer, not to opposite beads of the hexlet as in the two-dimensional equation, but to the two on either side of the one to which the bend, $\delta$, has been assigned. Hence we have

$\varphi_{+}+\varphi_{-}=\sigma+\delta$; or $\varphi_{N+1}+\varphi_{N-1}=\sigma+\varphi_{N}$; or $\varphi_{3}=\sigma+\varphi_{2}-\varphi_{1}$,

where $\varphi_{N+1}, \varphi_{N}$ and $\varphi_{N-1}$ refer to the bends of any three consecutive beads. From this, any two consecutive beads being known, all the others can be written down at once, when it will be found, quite unexpectedly I think, that this simple relation conceals a periodic function, with a perjod of six, or $\varphi_{N+6}$ $=\varphi_{N}$. Thus the six beads have values, in terms of those of any two consecutive beads, $\varphi_{1}$ and $\varphi_{2}$, (1) $\varphi_{1}$; (2) $\varphi_{2}$; (3) $\varphi_{3}=\sigma+\varphi_{2}-\varphi_{1}$; (4) $\varphi_{4}=\sigma+\varphi_{3}-\varphi_{2}=2 \sigma$ $-\varphi_{1}$; (5) $\varphi_{5}=\sigma+\varphi_{4}-\varphi_{3}=2 \sigma-\varphi_{2}$; (6) $\varphi_{8}=\sigma+$ $\varphi_{5}-\varphi_{4}=\sigma+\varphi_{1}-\varphi_{2} ;(7) \varphi_{7}=\sigma+\varphi_{6}-\varphi_{5}=\varphi_{1}$.

It will be seen that the sum of the bends of each opposite pair remains constant at $2 \sigma$, though any value $\delta$ between limits was given to the one bend arbitrarily fixed. The hexlet in fact is free to revolve round the trio without loss of serial contact, or separation of its several beads each from contact with all three of the trio. Similarly any given hexlet may be fixed and the trio varied through a definite cycle in which $\sigma$ remains unchanged but the three individual bends vary interdependently.
The primary hexlet of the bowl of integers is free to revolve in its tri-spherically sided annulus without change of grade, because exceptionally all its beads are identical, and this property is not due to two of the spheres of the trio being equal, but derives from the sum of their radii being equal to that of the bowl. Using Roman letters for radii, $A$ and $B$ may have any values so long as they sum to $C$, or using Greek letters for bends, so long as $1 / \alpha+1 / \beta+1 / \gamma=0$. When we introduce this value for $\gamma$ into the surd of equation (I) for four circles in mutual contact $\alpha \beta+\alpha \gamma+\beta \gamma$, it vanishes and $\varphi_{+}=\varphi_{-}=\sigma$. When we introduce these values for $\gamma$ and $\delta, \delta$ being the $\varphi$ of equation (1) when $\varphi_{+}=\varphi_{-}$, into equation (2), its surd

$$
\begin{aligned}
& 6(\alpha \beta+\alpha \gamma+\alpha \delta+\beta \gamma+\beta \delta+\gamma \delta)-3\left(\alpha^{2}+\beta^{2}+\right. \\
& \left.\gamma^{2}+\delta^{2}\right), \text { or }(\varphi+-\varphi-)^{2},
\end{aligned}
$$

also becomes zero, and $\varphi_{+}=\varphi_{-}$. That involves that all the six spheres of the hexlet are identical, because the opposite members as well as those on each side of them are all equal.

In conclusion, one point of really fascinating historical interest emerges. The Greeks, in their study of the $\alpha \rho \beta \eta \lambda \circ \varsigma$ or "shoemaker's knife", obtained by pure geometry the radius of the fourth circle that will make mutual contact with a mutually tangent trio for which $A+B=C$, whatever the ratio $A: B$. The proposition from the Arabic "Liber Assumptorum" will be found in full with notes in Sir Thomas Heath's "Works of Archimedes", p. 307 (Camb. Univ. Press, 1897). In the actual figure by which the proof was demonstrated $A / B$ was $3 / 2$, which gives for the radius in question $6 / 19$, in terms of $C$ as unity, when, therefore, $A$ is $3 / 5$ and $B$ is $2 / 5$. Since $\varphi=\sigma$,

$$
\varphi=5 / 3+5 / 2-1=19 / 6 !
$$

It is curious this should have remained unnoticed for thousands of years. Possibly when a mental arithmetic of reciprocals becomes as natural as that of number we may hope to learn the real secret of the circle and the sphere.

\section{Climatic Discomfort}

$\mathrm{M}^{\mathrm{s}}$ R. W. F. TYLER, an engineer interested in meteorology, was led to make an investigation into the connexion between climatic discomfort and the state of the atmosphere in regard to temperature and humidity. A summary of his work is to be found in a reprint of a paper read before Section $J$ (Psychology) of the British Association at Blackpool.

Mr. Tyler began his work at Shanghai more than thirty years ago. He was helped by the late Father Froc, then director of Siccawei Observatory, Shanghai, and by twelve observers-well-educated, intelligent men, one gathers-who during the month of August 1902 made an observation at every noon of their sensation of discomfort from the heat and dampness while sitting quietly in their offices, cooled by the punkah or fan to which they were accustomed. Their sensations were recorded on an arbitrary scale extending from I to 10 ; the numbers on the scale have been called hythers, hyther 10 being taken to represent an "unbearable condition". As the result of these determinations we have a very interesting diagram in which the hythers are shown as sloping, nearly parallel, slightly curved lines derived from plottings of the individual observations with temperatures as abscissæ and the differences between the dry and wet bulb thermometers as ordinates. The course of each hyther shows how much change in the depression of the wet bulb was required on an average to prevent any given change of temperature from altering the discomfort appropriate to that hyther.

One is tempted to criticize this fundamental diagram, on which a number of climatic studies have been based, on the grounds that the data are too meagre, that one individual differs greatly from another in response to atmospheric conditions, and that each varies according to health, state of mind and other influences, but Mr. Tyler surely deserves credit for trying to bring more precision into the psychology of hot climates, and his work should be 